\title{
Mars 520-d mission simulation reveals protracted crew hypokinesis and alterations of sleep duration and timing
}

\author{
Mathias Basner ${ }^{a, 1,2}$, David F. Dinges ${ }^{a, 1,2}$, Daniel Mollicone ${ }^{b}$, Adrian Ecker ${ }^{a}$, Christopher W. Jones ${ }^{a}$, Eric C. Hyder ${ }^{a}$, \\ Adrian Di Antonio ${ }^{a}$, Igor Savelev ${ }^{c, d}$, Kevin Kan ${ }^{b}$, Namni Goel ${ }^{\mathrm{a}}$, Boris V. Morukov ${ }^{\mathrm{e}}$, and Jeffrey P. Sutton ${ }^{\mathrm{d}, \mathrm{f}, 2}$ \\ aDivision of Sleep and Chronobiology, Unit for Experimental Psychiatry, Department of Psychiatry, Perelman School of Medicine, University of Pennsylvania, \\ Philadelphia, PA 19104; bulsar Informatics, Inc., Philadelphia, PA 19104; ' Human Performance and Engineering Division, Wyle, Houston, TX 77058; ${ }^{\mathrm{b}}$ National

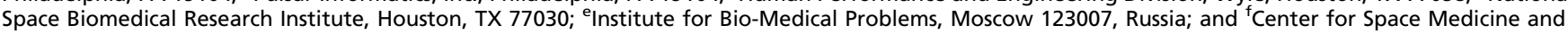 \\ Department of Medicine, Baylor College of Medicine, Houston, TX 77030
}

Edited by Joseph S. Takahashi, Howard Hughes Medical Institute and University of Texas Southwestern Medical Center, Dallas, TX, and approved November 27, 2012 (received for review July 31, 2012)

\begin{abstract}
The success of interplanetary human spaceflight will depend on many factors, including the behavioral activity levels, sleep, and circadian timing of crews exposed to prolonged microgravity and confinement. To address the effects of the latter, we used a highfidelity ground simulation of a Mars mission to objectively track sleep-wake dynamics in a multinational crew of six during $\mathbf{5 2 0} \mathrm{d}$ of confined isolation. Measurements included continuous recordings of wrist actigraphy and light exposure (4.396 million $\mathrm{min}$ ) and weekly computer-based neurobehavioral assessments $(n=888)$ to identify changes in the crew's activity levels, sleep quantity and quality, sleep-wake periodicity, vigilance performance, and workload throughout the record-long $\mathbf{1 7}$ mo of mission confinement. Actigraphy revealed that crew sedentariness increased across the mission as evident in decreased waking movement (i.e., hypokinesis) and increased sleep and rest times. Light exposure decreased during the mission. The majority of crewmembers also experienced one or more disturbances of sleep quality, vigilance deficits, or altered sleep-wake periodicity and timing, suggesting inadequate circadian entrainment. The results point to the need to identify markers of differential vulnerability to hypokinesis and sleepwake changes during the prolonged isolation of exploration spaceflight and the need to ensure maintenance of circadian entrainment, sleep quantity and quality, and optimal activity levels during exploration missions. Therefore, successful adaptation to such missions will require crew to transit in spacecraft and live in surface habitats that instantiate aspects of Earth's geophysical signals (appropriately timed light exposure, food intake, exercise) required for temporal organization and maintenance of human behavior.
\end{abstract}

sleep-wake regulation | astronaut

$\mathbf{T}$ he success of human interplanetary spaceflight, which is anticipated to be in this century, will depend on the ability of spacefarers to remain confined and isolated from Earth much longer than previous missions or simulations, while maintaining the intensity and timing of behavioral activity necessary to accomplish the mission and mitigate the effects of microgravity. A total of four people have spent $>1 \mathrm{y}$ in space, with the record of 437 consecutive days on the Mir space station set by Valery Polyakov. The longest Earth-based spaceflight simulation involved four Russians confined in connected hyperbaric chambers for 240 consecutive days. Antarctic winter-over missions have extended up to $363 \mathrm{~d}$. Prediction of how prolonged confinement affects activity levels and sleep-wake dynamics of space explorers is needed to inform spacecraft habitability requirements, crew selection, and behavioral countermeasures during interplanetary missions (1-3). To address this need, we obtained objective neurobehavioral data on the activity patterns of a multinational, culturally diverse crew of six males with backgrounds in engineering, medicine, physiology, and space training, who participated in a high-fidelity ground simulation of a 520-d mission to Mars. Ecological validity of the simulation included a spaceship-like habitat; continuous isolation from Earth's environment; realistic mission activities; a midmission landing on a simulated Mars surface; accurate mission duration and timeline; operations between crew and mission controllers; communication delays inherent in interplanetary travel; limited consumable resources; exercise equipment for physical fitness; diurnal weekly work schedule; crew control of habitat lighting; and video monitoring of crew in habitat common areas. The simulation was developed and operated by the Institute for Bio-Medical Problems (IBMP) of the Russian Academy of Sciences. Photos of the simulation facility and detailed descriptions of the crew, mission timeline, and work-rest schedule are provided in SI Appendix, Fig. S1 and Tables S1-S3).

\section{Results}

Movement acceleration of crewmembers was continuously recorded at a 1-min resolution throughout the 520-d mission to track the intensity and duration of active wakefulness, rest, and sleep by using validated wrist actigraphy devices that also recorded light intensity (SI Appendix, SI Text and Fig. S2). The result was $4.396 \times 10^{6} \mathrm{~min}$ of data constituting $98.0 \%$ of time in mission. Behavioral alertness of the crew was probed twice weekly by using psychomotor vigilance test (PVT-B) performance $(4,5)$ with simultaneous video of the face. Weekly crew ratings were obtained for workload, tiredness, and sleep quality (SI Appendix, SI Text).

Changes in Crew Rest-Activity Dynamics During the Mission. Profiles of the crew's time and movement intensity in discrete behavioral states indicated increasing sedentariness across the mission. Time spent in active wakefulness per $24 \mathrm{~h}$ dropped sharply during the first $3 \mathrm{mo}$, then more gradually across the next 13 mo

Author contributions: M.B., D.F.D., D.M., A.E., C.W.J., E.C.H., A.D.A., I.S., K.K., N.G., B.V.M., and J.P.S. designed research; M.B., D.F.D., D.M., A.E., C.W.J., E.C.H., A.D.A., I.S., K.K., N.G., B.V.M., and J.P.S. performed research; M.B., D.F.D., D.M., C.W.J., E.C.H., A.D.A., K.K., and N.G. analyzed data; and M.B., D.F.D., C.W.J., E.C.H., A.D.A., N.G., and J.P.S. wrote the paper

The authors declare no conflict of interest.

This article is a PNAS Direct Submission.

Data deposition: The data reported in this paper are deposited in a Microsoft Excel file. To access the data: i) Go to the www.med.upenn.edu/uep/user_documents/PNAS_Basner_et_al. xlsx. ii) When the Save File window appears, choose a destination location to save the file.

${ }^{1}$ M.B. and D.F.D. contributed equally to this work

${ }^{2}$ To whom correspondence may be addressed. E-mail: dinges@mail.med.upenn.edu, basner@mail.med.upenn.edu, or jps@bcm.edu.

This article contains supporting information online at www.pnas.org/lookup/suppl/doi:10. 1073/pnas.1212646110/-/DCSupplemental. 
of the mission (Fig. 1A). In the final 20 mission days, wake time and intensity rose sharply, whereas sleep and rest times decreased sharply relative to two preceding sequential 60 -d periods $(P<0.002)$. Scheduled workload and crew ratings of workload did not increase in these final $20 \mathrm{~d}$. Mission managers indicated that the increased activity of the crew was due to their psychological anticipation of mission end.

Actigraphically estimated mean sleep per 24-h day throughout the mission was $7.39 \mathrm{~h}(\mathrm{SE}=0.20 \mathrm{~h}$; SI Appendix, Table S5A). When corrected by $-26.4 \mathrm{~min}(0.44 \mathrm{~h})$ based on our validation study of the Actiwatch algorithm (SI Appendix, SI Text and Fig. $\mathrm{S} 2$ ), mean mission sleep duration is estimated to have been $6.95 \mathrm{~h}$. Because the correction estimate has a 16.8-min 95\% confidence interval, all results reported are for uncorrected actigraphy values. Sleep and rest times showed an inverse pattern to wake time throughout the mission, increasing across the mission until the final 2 mo (Fig. 1A). Analyses by mission quarter (MQ; i.e., 130 -d periods) revealed a $7.0 \%$ decrease in active wakefulness across the mission, equivalent to 1.12 -h less active waking per day per crewmember in the last compared with the first MQ (Fig. $1 B ; P<0.0001)$.

Sleep time increased by $8.4 \%$ across the mission, equivalent to 0.59 -h more sleep per day per crewmember in the last compared with the first MQ (Fig. $1 C ; P<0.0001$ ). Rest time increased by $50.4 \%$ across the mission, equivalent to 0.54 -h more rest per day per crewmember in the last relative to the first MQ (Fig. $1 D ; P<$ $0.0001)$. The findings were confirmed by analyses confined to only the nocturnal (SI Appendix, Fig. S3A) or only the diurnal portion of each day (SI Appendix, Fig. S3B), which indicates that the time of day that active wake, rest, and sleep were obtained, did not alter the progressive sedentariness of the crew across the mission. For $90 \%$ of mission time awake, crewmembers were exposed at the wrist to light intensity of $<177$ lux (lx). The intensity of ambient light the crew was exposed to while actively awake during the mission declined by $25.6 \%$ from a mean of $104.8 \mathrm{~lx}(\mathrm{SE}=4.9)$ to a mean of $78.0 \mathrm{~lx}(\mathrm{SE}=8.5)$ across $\mathrm{MQs}$
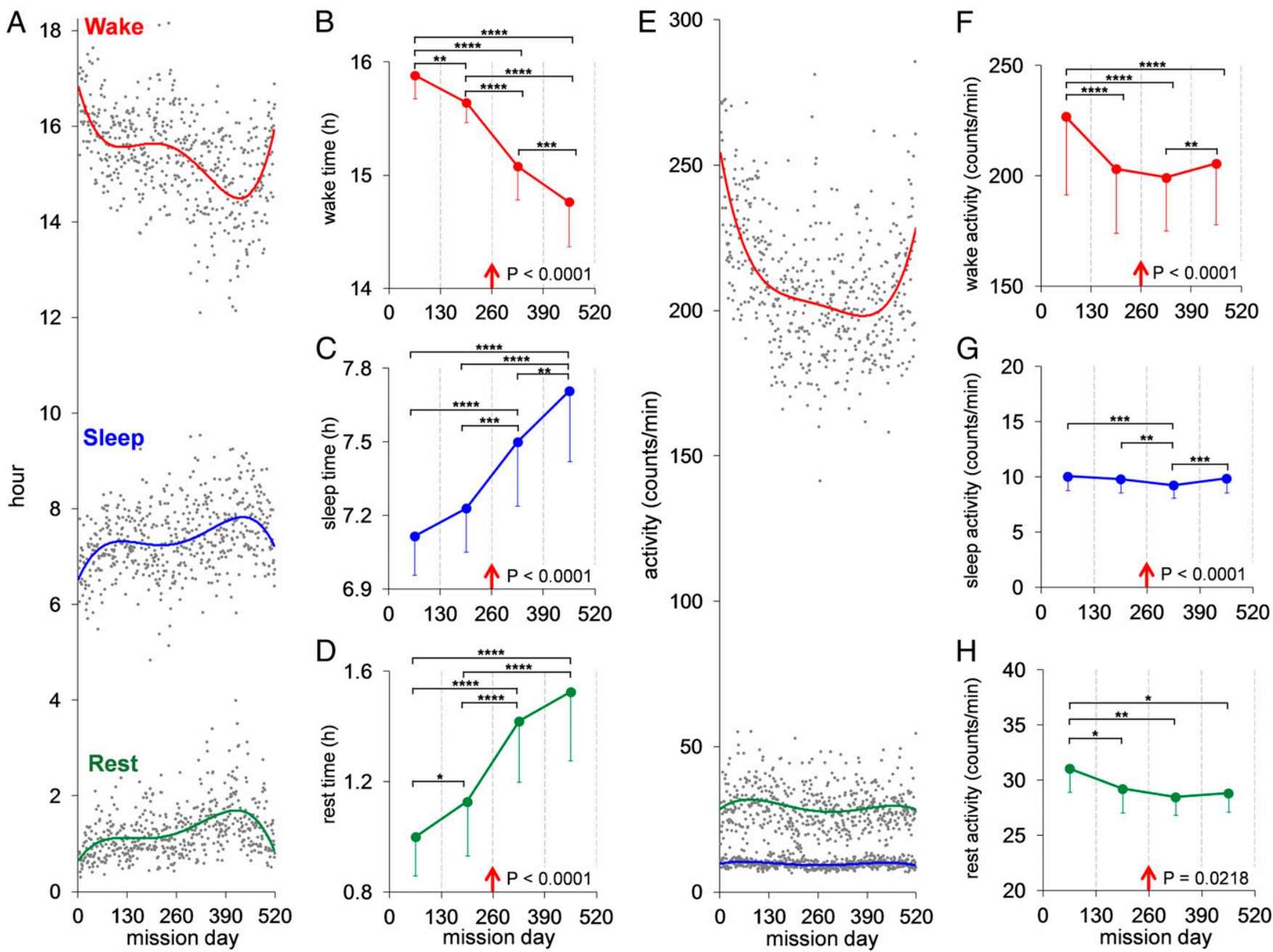

Fig. 1. Activity profiles of crewmembers measured by continuously worn wrist actigraphs throughout the 520 - $d$ simulated mission. (A) The crew's daily mean (gray points) time (hours) spent in active wakefulness (red trend line), sleeping (blue trend line), and resting (green trend line) across the mission (for information on statistical analyses, see SI Appendix, SI Text). (B-D) The crew's mean (SE) time in each behavioral state for each consecutive 130-d MQ (red arrow shows simulated midmission landing on Mars). There was a systematic decrease across MQs in active wakefulness ( $B$ ) and systematic increases in both sleep time $(C)$ and waking rest time $(D)$. F test $P$ values for these effects are shown in each graph; post hoc tests between $\mathrm{MQs:} * P<0.05, * \star P<0.01, * * \star P<0.001$, $\star * * * P<0.0001$. (E) The crew's daily mean (gray points) intensity of activity (counts per min) across the mission during active wakefulness (red trend line), sleep (blue trend line), and rest (green trend line). $(F-H)$ The crew's mean (SE) intensity of activity in each state by MQ. Activity levels declined in MQ 2 and much of MQ 4 relative to the first $M Q$, but rose in the final $20 \mathrm{~d}$ of the last $\mathrm{MQ}$ ( $F$ test $P$ values are shown in $F-H)$. The sharp increases in active wake time $(A)$ and intensity $(E)$ in the final 20 mission days, and the commensurate sharp decreases in sleep and rest times $(A)$, were significantly different from mean values for these variables relative to two sequential 60 - $d$ periods immediately before the final $20 \mathrm{~d}$ of the mission $(P<0.002)$. 
$(P<0.0001)$ and increased slightly during rest (SI Appendix, Fig. S3C).

Integrated peak acceleration change per second was used to evaluate movement intensity during each behavioral state. Active waking underwent a steep decline in movement intensity during the first $\mathrm{MQ}$, followed by a more gradual decline for $1 \mathrm{y}$ and a sharp rise in the final 20 mission days (Fig. $1 E$ ). Analyses of this hypokinesis by MQ confirmed reliability of the trend (Fig. $1 F$; $P<0.0001)$. The activity changes during the mission mirrored the crew's ratings of workload, which were highest in the first MQ and lower in subsequent MQs (SI Appendix, Fig. S4A; F test, $P<0.0001)$. Crew sleep durations averaged $<6.78 \mathrm{~h}(\mathrm{SE}=0.19 \mathrm{~h})$ per day during the first $40 \mathrm{~d}$ of the mission, which was lower than during all subsequent $40-\mathrm{d}$ mission periods $(P<0.05)$. This value is close to actigraphically recorded sleep times of astronauts on US Space Shuttle missions (6) and International Space Station expeditions, where work tempos have historically been high Sleep durations chronically at $<7 \mathrm{~h}$ per day result in cumulative neurobehavioral performance deficits across days $(7,8)$, making chronic partial sleep loss a risk to optimal performance in space (3).

The work-rest schedule throughout the mission was $5 \mathrm{~d}$ on and $2 \mathrm{~d}$ off. The decrease in active wake and increase in sleep and rest times as the mission progressed occurred on both work and rest days (F tests, $P<0.0001$ ). Other than the first $40 \mathrm{~d}$ of the mission, the only transient interruption of increasing sleep time with mission duration involved the 80 -d period before and including the midmission simulated Mars surfacing, when mean sleep duration declined from $7.33 \mathrm{~h}(\mathrm{SE}=0.15)$ to $7.13 \mathrm{~h}(\mathrm{SE}=$ $0.22)(P=0.0379)$, and crew ratings of tiredness increased $(P=0.0108)$.

Collectively, the crew did not manifest cumulative deficits in PVT-B vigilance performance. Crew daily sleep time increased from a mean of $7.12 \mathrm{~h}$ in MQ 1 to $7.71 \mathrm{~h}$ in MQ 4 (Fig. $1 C$; F test, $P<0.0001$ ), which included reliable increases in sleep duration from MQ 2 to 3 (post hoc test, $P<0.001$ ) and from MQ 3 to 4 $(P<0.01)$, when workload ratings were not changing (SI Appendix, Fig. S4A). The cumulative effect of the increasing time spent asleep over the 1.42-y-long mission was substantial. In total, the crew obtained $673 \mathrm{~h}$ more sleep in the second half of the mission relative to the first half (SI Appendix, Table S5B). Consistent with the increased sleep time, their average normal PVT-B response speed further improved, and their already low rate of vigilance lapses further decreased during the second half of the mission (F tests, $P<0.0001$; SI Appendix, Fig. S5 $A$ and $B$ ). Thus, the added sleep in the latter half of the mission likely benefited behavioral alertness and psychomotor speed, and it may have occurred as a result of confinement, monotony, and habitat characteristics, which included relatively low light levels $(<130 \mathrm{~lx}$; SI Appendix, Fig. S3C) and privacy from monitoring cameras available only in areas for personal hygiene and sleeping quarters.

Variation Among Crewmembers in Sleep-Wake Activity. Cumulative functions were used to evaluate the degree to which changes in activity states across the mission reflected variation in crewmember adaptation to the mission. These functions revealed substantial differences among crewmembers that were unrelated to their roles, responsibilities, or workload ratings. For example, crewmember $d$ maintained the highest wake activity level across the mission (Fig. $2 A$ ) but also one of the highest sleep amounts (Fig. $2 B$ ) and the most frequent ratings of good sleep quality (SI Appendix, Table S6A). In contrast to the rest of the crew, he had a very low rest time (Fig. $2 C$ ) and a PVT-B performance error rate that was low (Fig. $2 D$ ). His data illustrate that high wake activity levels and adequate sleep to maintain alertness are not incompatible in long-duration mission confinement.

Crewmember $f$ had one of the lowest wake activity levels across the mission (Fig. $2 A$ ). He also had the lowest sleep amount
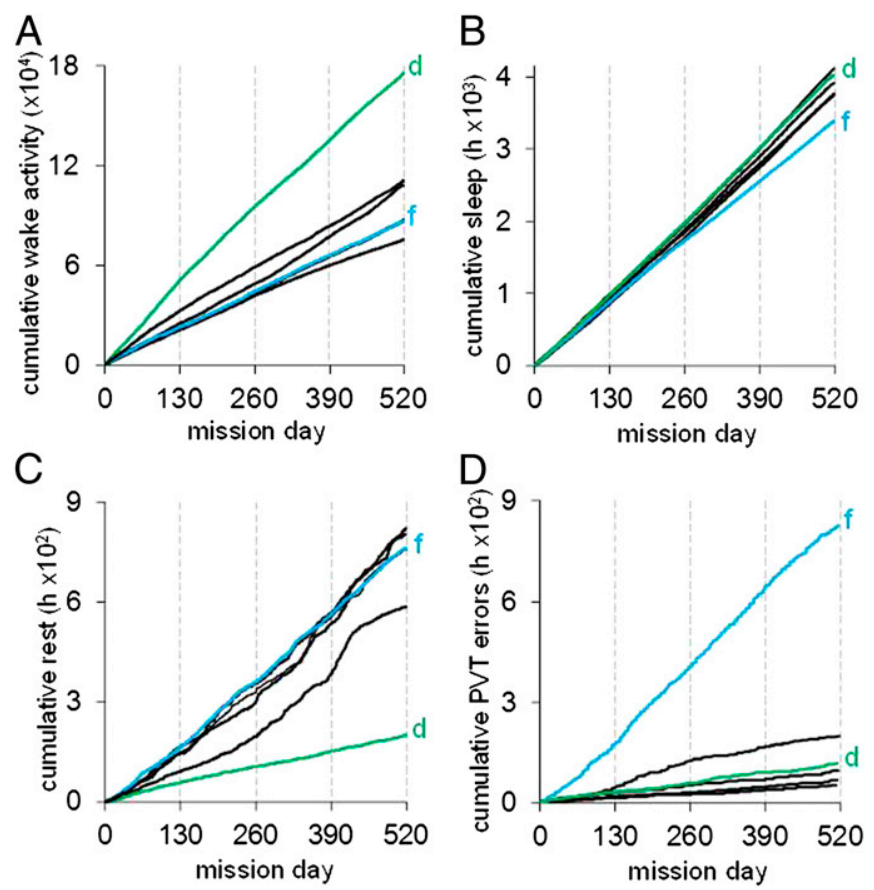

Fig. 2. Cumulative functions over $520 \mathrm{~d}$ of mission confinement for each crewmember's waking activity levels $(A)$, time spent in sleep $(B)$ and rest $(C)$, and PVT-B error rate $(D)$. Examination of data from crewmembers $d$ and $f$ illustrate the interindividual differences among the crew in reaction to the prolonged mission confinement.

(Fig. $2 B$ ) among the crew throughout the mission (mean $=6.54 \mathrm{~h}$, $\mathrm{SE}=0.04$; SI Appendix, Table S5A) and the most frequent ratings of poor sleep quality (SI Appendix, Table S6A). He was comparable in rest time to most of the crew (Fig. $2 C$ ). He had a much higher PVT-B performance error rate than other crewmembers (Fig. 2D). He also had the majority of PVT-B facial videos displaying sleepiness and the most frequent ratings of difficulty performing the PVT-B (SI Appendix, SI Text). PVT-B performance is highly sensitive to acute and chronic sleep loss and is devoid of a learning curve $(4,9)$. The performance of crewmember $f$ was consistent with his experiencing chronic partial sleep deprivation throughout the mission.

There were also differences among crew in sleep-wake timing and periodicity. Operations were organized around 24-h clock time with a daily 8.5-h nocturnal sleep period (SI Appendix, Table S3). The crew had control over habitat lighting, food intake, physical exercise, and other factors that can promote circadian entrainment (10), but they were not exposed to Earth's geophysical light-dark cycle. The endogenous period of the human circadian pacemaker regulating sleep-wake timing averages $24.18 \mathrm{~h}(11)$, but it can be entrained to a 24.0 -h period by certain synchronizers, the most important being ambient light. The appropriate phase, intensity, duration, and spectral characteristics of light can promote entrainment and thereby stabilize the timing of behavioral states relative to environmental time, ensuring daytime wakefulness and sleep at night (12). Measurements of light in the crew facility revealed a spectral power distribution consistent with fluorescent lighting (SI Appendix, Fig. S6) with low intensity in the 446- to 477-nm wavelength region of the photon spectrum, which is the most potent region for synchronizing or phase-shifting circadian rhythms of sleep and waking $(13,14)$ and for promoting sleep timing in polar darkness $(15)$. A separate experiment sponsored by the European Space Agency involved adding blue-light exposure late (days 439-499) in the mission (SI Appendix, SI Text). 
Spectrographic analyses of actigraphy data across the mission were conducted for each crewmember to evaluate the extent to which 24-h timing of sleep-wake cycles was maintained during the mission (Fig. 3). Four crewmembers had a monophasic nocturnal sleep pattern throughout the mission with a 24-h sleepwake cycle (Fig. $3 C-F$ ). Crewmember $a$ manifested a split-sleep pattern (i.e., nocturnal anchor sleep plus a diurnal nap), which became more pronounced in the latter half of the mission as evidenced by a $50.8 \%$ increase in diurnal sleep (Fig. $3 A$ ). Despite this increasing displacement of sleep from the nocturnal to the diurnal portion of the day, a 24-h periodicity of sleep timing was evident for crewmember $a$ because a major sleep episode always occurred nocturnally.

In contrast to other subjects, crewmember $b$ had a sleep-wake cycle with a dominant period of $24.98 \mathrm{~h}$, which lengthened across MQs from 24.72 to $25.06 \mathrm{~h}$ (Fig. 3B). This prolonged sleep-wake period is beyond the endogenous free-running circadian period found in healthy adults (11), but is very similar to periods observed in older, shorter-duration circadian isolation protocols (17) and polar studies (18), wherein subjects had access to roomlight exposure before circadian temperature minimum. A number of factors may contribute to a prolonged sleep-wake cycle during confinement and isolation, including exercise (19) and light exposure at sensitive portions of the circadian-phase response curve for each zeitgeber (11). The circadian system is sensitive to even low levels of light before body temperature minimum (20), which can induce phase delays in molecular mechanisms of entrainment (21), suppress melatonin secretion (13), and induce longer sleep-wake periods (22). Examination of light-exposure data revealed that crewmember $b$ was awake later at night and exposed to light at times that may have contributed to repeated phase delays of his sleep-wake cycle $(23,24)$. This nocturnal room-light exposure during a sensitive phase for circadian delays began in the first $30 \mathrm{~d}$ of the mission (SI Appendix,
A

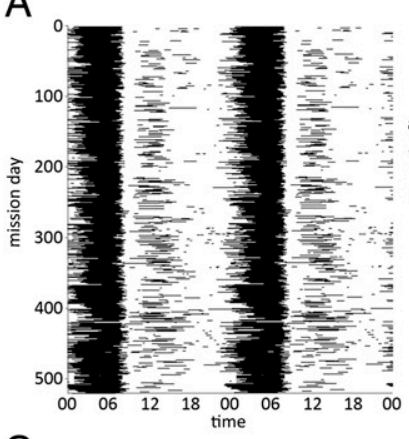

C

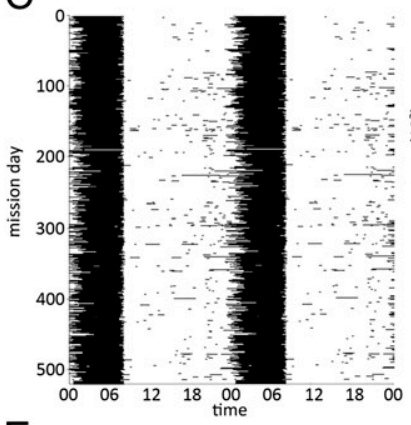

E

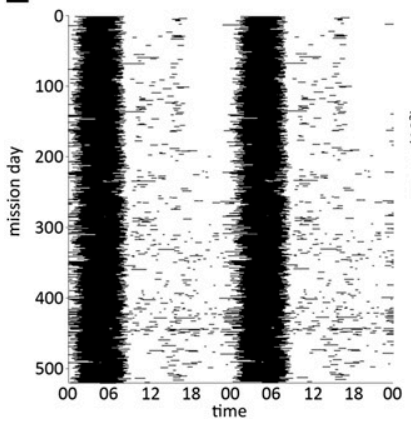

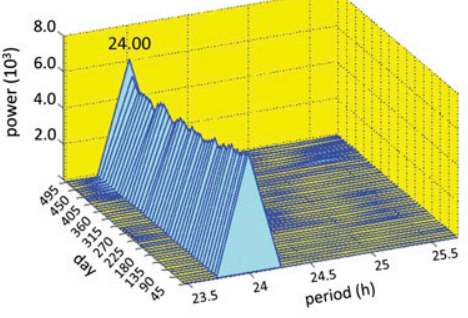

B
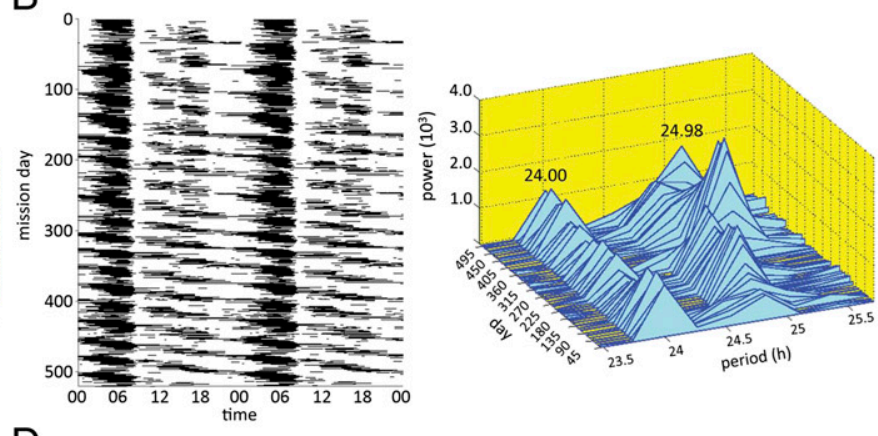

D
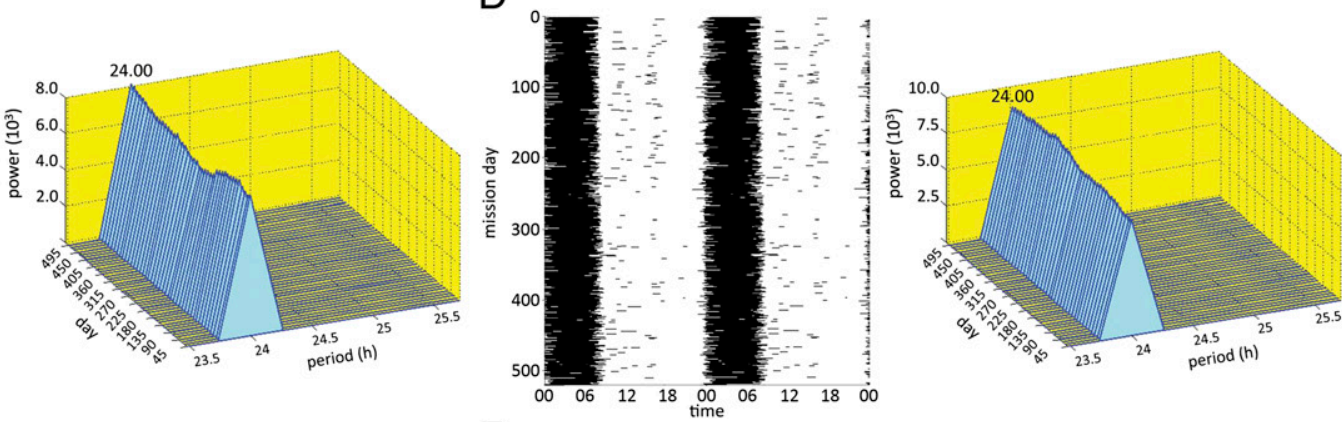

$\mathrm{F}$

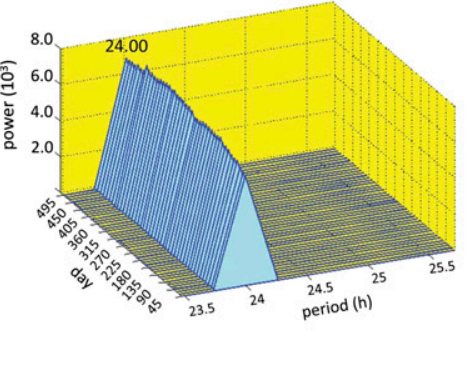

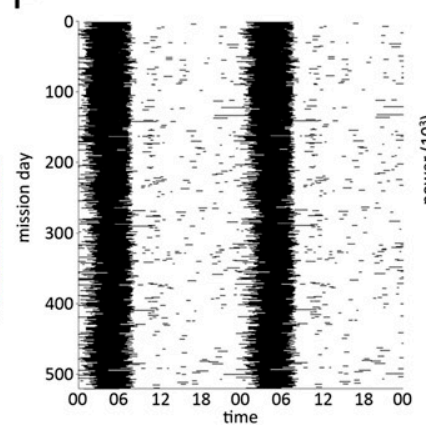

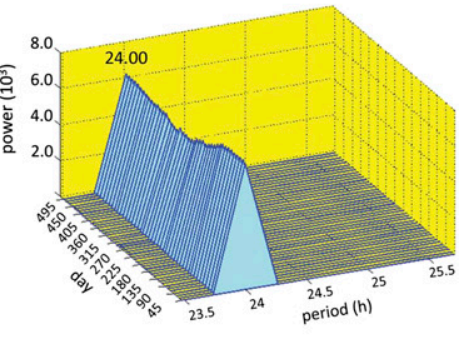

Fig. 3. Double raster plots of sleep (black) and wake (white) and spectral plots (blue and yellow) from actigraphically derived sleep and waking throughout the 520-d mission for crewmembers $a, b, c, d$, e, and $f(A-F$, respectively). Rest was classified as wake for these analyses. Spectral analyses to evaluate sleepwake periodicity were performed on 1-min actigraphic epochs based on the power spectral density by using the periodogram method (16), multiplying the data with a 90-d rectangular window and taking the squared magnitude of the discrete time Fourier transform. The peak frequency was estimated by a 3 point quadratic interpolation based on the log-magnitudes of the periodogram at the frequency corresponding to the maxima in the periodogram and the two neighboring points. Spectrogram plots were derived from the 90 -d window moved in increments of $10 \mathrm{~d}$ across the mission (SI Appendix, SI Text). As is evident in the double-raster and spectral plots, all crewmembers except $b$ had a predominant 24-h sleep-wake periodicity. Crewmember $b$ had a sleep-wake period that varied between 24.72 and $25.06 \mathrm{~h}$ across the mission, increased with time in mission, and averaged $24.98 \mathrm{~h}$ for the entire mission. The smaller $24-\mathrm{h}$ peak seen in the spectrogram of crewmember $b$ was due to his daily attendance at breakfast between 08:00 and 10:00 each morning (SI Appendix, Table S3). 
Fig. S7 $A$ ) and occurred periodically thereafter as the sleep-wake cycle lengthened to near $25 \mathrm{~h}$ (SI Appendix, Fig. S7B).

The near 25-h sleep-wake period of crewmember $b$ (Fig. $3 B$ ) and the biphasic sleep pattern of crewmember $a$ (Fig. $3 A$ ) were associated with more mission days in which their total sleep times was $>10 \mathrm{~h}(12.6 \%$ and $12.2 \%$, respectively) relative to all other crewmembers (mean $=1.7 \%)$. They were the only two crewmembers who showed average delayed sleep onset times of $\sim 2 \mathrm{~h}$ or more during the first month of the mission relative to their premission averages- $3 \mathrm{~h} 22 \mathrm{~min}$ for crewmember $b$ and $1 \mathrm{~h}$ 59 min for crewmember a (SI Appendix, SI Text and Table S4). This result is consistent with both crewmembers experiencing delayed sleep-phase syndrome or non-24-h sleep-wake syndrome (25). The longer-duration sleep episodes and increased temporal displacement of sleep resulted in these two crewmembers being asleep when other crewmembers were awake (or vice versa) a total of $2,498 \mathrm{~h}$, or $20.1 \%$ of the mission (SI Appendix, Fig. S7C). Such unplanned temporal desynchrony among crewmembers has the potential to pose a challenge for effective crew coordination during long-duration spaceflight.

\section{Discussion}

A majority (four of six) of the crewmembers in this record-long, high-fidelity, simulated space mission confinement experienced one or more of the following problems: disrupted sleep-wake periodicity $(n=1)$, increased displacement of sleep into the diurnal period $(n=2)$, performance deficits associated with chronic partial sleep deprivation $(n=1)$, and frequent reductions in perceived sleep quality $(n=2)$. Spectral plots (Fig. 3) and cumulative functions (Fig. 2) indicate that these problems occurred early in the mission and persisted unabated. Such individual differences in disturbances of sleep-wake regulation are similar to those identified in winter-over polar expeditions (15, 26), which are often considered analogs for the study of behavioral reactions to the prolonged isolation of spaceflight (27-30). The fact that sleep-wake disruptions occur during confinement and isolation in some individuals more than others is consistent with increasing evidence that there are phenotypic and genotypic differences in vulnerability to both alterations of sleep and the resulting neurobehavioral consequences (31-37). This differential vulnerability has led to a search for predictive biomarkers of the effects of sleep loss (38), which would be useful for managing sleep-wake regulation during exploration spaceflight (39).

The progressive sedentariness of the crew that was evident through increased sleep and rest times and the decreased active wakefulness with time in mission support the view that ecological variables can be determinants of human sleep duration (40). It is suggestive of behavioral aspects of torpor, which historically refers to lethargy (41) but more recently has been used to define metabolic or body temperature changes characteristic of heterothermic mammals and birds (42). The concept of behavioral torpor as sedentariness is consistent with the increases in sleep reported in some migratory birds and other animals living in confinement or during winter photoperiods $(43,44)$.

The hypokinesis and behavioral torpor during the 520-d simulated exploration mission, the sleep loss induced by critical periods of high workload early in the mission, and the common and persistent disturbances of sleep-wake behaviors throughout the mission highlight the importance of preventing these conditions in exploration missions. There is a need for novel space exploration habitats and crew activity schedules that mimic the biological potency of Earth's geophysical cycle through both photic $(22,45)$ and nonphotic $(10,19,46)$ synchronizers to promote circadian entrainment and the temporal optimization of behavioral states during prolonged spaceflight. These needs extend to circadian adjustment for work in near-circadian environments, such as the Martian solar day (i.e., 24.67 h) (47). A balance must be struck during human exploration of space between the critical need for adequate time for sleep and rest and the need to maintain activity levels for physical and physiological fitness. This balance is especially important given the deleterious effects of prolonged microgravity on the musculoskeletal, cardiovascular (48), and other systems (49) and the requirement to sustain fitness to work effectively, avoid injury, and successfully accomplish the mission.

Our findings also have implications for the increasing prevalence of sleep and circadian rhythm disorders among humans living on Earth in industrialized societies, with limited exposure to natural geophysical signals, widespread sedentary activities, and primarily artificial light exposure. There is considerable population evidence that work schedules (50), alarm clocks (51), television programming times (51), and cultural time shifts, such as school start times (52) and daylight savings time (53), contribute to sleep restriction and a discrepancy between circadian and social clocks (i.e., social jetlag), both of which have been linked to obesity (54). The essential need for humans to maintain sleep-wake activity cycles synchronized to the circadian biology that temporally coordinates human health and behavior appears to be as important on Earth as it will be en route to Mars.

\section{Methods}

The State Scientific Center of the Russian Federation-IBMP of the Russian Academy of Sciences (RAS) performed the 520-d simulated mission. Crewmembers signed informed consents approved by the Institutional Review Board of the University of Pennsylvania.

Wrist actigraphy (Actiwatch Spectrum; Philips/Respironics) for assessing sleep-wake activity (55) was worn by crewmembers throughout the $520 \mathrm{~d}$. Both average light intensity and movement-induced accelerations at the wrist were recorded in 1-min epochs. Activity data were classified into active wake, sleep, or waking rest using Respironics Actiware (Version 5.59.0015). A separate validation study that we conducted of the Actiwatch state scoring algorithm established its detection sensitivity for sleep at $97.0 \%$, its specificity for wakefulness at $96.2 \%$, and overall accuracy of the Actiwatch algorithm at $96.4 \%$ (SI Appendix, SI Text and Fig. S2). A total of 4,396,333 min of activity was collected in the 520-d mission, which was $98.02 \%$ of the total possible. Actigraphy was also used to evaluate the intensity of activity for each $24-\mathrm{h}$ period during the mission and in each of the three states.

Spectrographic analyses of actigraphy data were performed on 1-min epochs to determine the predominant periodicity of sleep-wake timing for each subject. Power spectra of the sleep-wake time series were estimated by using the periodogram method (16) of multiplying the data with a 90-d rectangular window and taking the squared magnitude of the discrete time Fourier transform.

Behavioral alertness was assessed by using psychomotor vigilance performance (refs. 4, 9, and 56; SI Appendix, SI Text) on a 3-min test (PVT-B) that was obtained weekly (once in the morning, once in the evening) by computer (4), with $100 \%$ complete data acquisition ( $n=888$ tests). Facial videos were recorded at 30 frames per second during each PVT-B and evaluated for slow eyelid closures indicative of sleepiness (57). Immediately before or after each PVT-B test, crewmembers completed computerized scales that included $100-\mathrm{mm}$ visual analog scales with the following binary anchors: good/poor sleep quality (morning only), high/low workload (evening only), and high/ low tiredness (evening only). Data acquisition for these subjective ratings was $100 \%(n=444)$.

Mixed-model ANOVAs (Proc Mixed; Version 9.3; SAS Institute) with a random intercept for crewmembers and unstructured covariance were performed with 130-d MQs as the explanatory variable. If a type 3 test indicated a significant MQ effect $(P<0.05)$, two-sided post hoc $t$ tests comparing individual MQs were performed. Fig. 1 and SI Appendix, Figs. S3-S5 graphically present these analyses. Significant findings of post hoc tests are indicated ( $\left.{ }^{*} P<0.05,{ }^{*} P<0.01, * \star P<0.001, * * \star \star P<0.0001\right)$. All statistical tests were two-tailed.

ACKNOWLEDGMENTS. The Mars 520-d simulation was developed by the Institute for Biomedical Problems of the Russian Academy of Sciences. We thank A. I. Grigoriev, I. B. Ushakov, E. P. Demin, and M. S. Belakovskiy for creating the simulation; the crew for participating in the study and providing the data; the crew of the 105-d pilot study for helping identify the optimal data acquisition techniques; E. Carota Orne for supporting the project; and D. Metaxas, C. Mott, R. Bartels, S. Arroyo, X. Yu, and J. Allen 
for aiding in data extraction. The research was supported by the National Space Biomedical Research Institute through National Aeronautics and Space Administration cooperative agreement NCC 9-58 and

1. Ball JR, Evans CH, eds (2001) Safe Passage: Astronaut Care for Exploration Missions (National Academy Press, Washington).

2. NASA, Human Research Roadmap: A Risk Reduction Strategy for Human Space Ex ploration. Available at http://humanresearchroadmap.nasa.gov/. Accessed July 31 2012.

3. Whitmire AM, et al. (2009) Risk of Performance Errors due to Sleep loss, Circadian Desynchronization, Fatigue and Work Overload: NASA Evidenced-Based Review (NASA, Washington), Human Research Program Requirements Document HRP-47052, Rev. C.

4. Basner M, Mollicone D, Dinges DF (2011) Validity and sensitivity of a brief psychomotor vigilance test (PVT-B) to total and partial sleep deprivation. Acta Astronaut 69(11-12):949-959.

5. Basner M, Rubinstein J (2011) Fitness for duty: A 3-minute version of the Psychomotor Vigilance Test predicts fatigue-related declines in luggage-screening performance. I Occup Environ Med 53(10):1146-1154.

6. Dijk DJ, et al. (2001) Sleep, performance, circadian rhythms, and light-dark cycles during two space shuttle flights. Am J Physiol Regul Integr Comp Physiol 281(5): R1647-R1664.

7. Van Dongen HPA, Maislin G, Mullington JM, Dinges DF (2003) The cumulative cost of additional wakefulness: dose-response effects on neurobehavioral functions and sleep physiology from chronic sleep restriction and total sleep deprivation. Sleep 26(2):117-126.

8. Belenky G, et al. (2003) Patterns of performance degradation and restoration during sleep restriction and subsequent recovery: A sleep dose-response study. J Sleep Res 12(1):1-12.

9. Lim J, Dinges DF (2008) Sleep deprivation and vigilant attention. Ann N Y Acad Sci 1129:305-322.

10. Fuller PM, Lu J, Saper CB (2008) Differential rescue of light- and food-entrainable circadian rhythms. Science 320(5879):1074-1077.

11. Czeisler CA, et al. (1999) Stability, precision, and near-24-hour period of the human circadian pacemaker. Science 284(5423):2177-2181.

12. Duffy JF, Wright KP, Jr. (2005) Entrainment of the human circadian system by light $J$ Biol Rhythms 20(4):326-338.

13. Brainard GC, et al. (1988) Dose-response relationship between light irradiance and the suppression of plasma melatonin in human volunteers. Brain Res $454(1-2)$ 212-218.

14. Dijk DJ, et al. (2012) Amplitude reduction and phase shifts of melatonin, cortisol and other circadian rhythms after a gradual advance of sleep and light exposure in humans. PLOS ONE 7(2):e30037.

15. Mottram V, Middleton B, Williams P, Arendt J (2011) The impact of bright artificia white and 'blue-enriched' light on sleep and circadian phase during the polar winter. J Sleep Res 20(1 Pt 2):154-161.

16. Hayes MH (1996) Statistical Digital Signal Processing and Modelling (John Wiley \& Sons, New York).

17. Aschoff J, Wever R (1962) Spontanperiodik des menschen bei ausschluß aller zeitgeber [Spontaneous periodicity in humans under exclusion of all zeitgebers]. Naturwissenschaften 49(15):337-342. German.

18. Kennaway DJ, Van Dorp CF (1991) Free-running rhythms of melatonin, cortisol, electrolytes, and sleep in humans in Antarctica. Am J Physio/ 260(6 Pt 2):R1137-R1144.

19. Barger LK, Wright KP, Jr., Hughes RJ, Czeisler CA (2004) Daily exercise facilitates phase delays of circadian melatonin rhythm in very dim light. Am J Physiol Regul Integr Comp Physiol 286(6):R1077-R1084.

20. Khalsa SB, Jewett ME, Cajochen C, Czeisler CA (2003) A phase response curve to single bright light pulses in human subjects. J Physio/ 549(Pt 3):945-952.

21. Schwartz WJ, Tavakoli-Nezhad M, Lambert CM, Weaver DR, de la Iglesia HO (2011) Distinct patterns of Period gene expression in the suprachiasmatic nucleus underlie circadian clock photoentrainment by advances or delays. Proc Natl Acad Sci USA 108(41):17219-17224

22. Lockley SW, Brainard GC, Czeisler CA (2003) High sensitivity of the human circadian melatonin rhythm to resetting by short wavelength light. J Clin Endocrinol Metab 88(9):4502-4505.

23. Gronfier C, Wright KP, Jr., Kronauer RE, Czeisler CA (2007) Entrainment of the human circadian pacemaker to longer-than-24-h days. Proc Natl Acad Sci USA 104(21): 9081-9086.

24. Zeitzer JM, Dijk DJ, Kronauer RE, Brown EN, Czeisler CA (2000) Sensitivity of the human circadian pacemaker to nocturnal light: Melatonin phase resetting and suppression. J Physiol 526(Pt 3):695-702.

25. Okawa M, Uchiyama M (2007) Circadian rhythm sleep disorders: Characteristics and entrainment pathology in delayed sleep phase and non-24-h sleep-wake syndrome. Sleep Med Rev 11(6):485-496. by the Institute for Experimental Psychiatry Research Foundation. The Friedkin Chair for Research in Sensory System Integration and Space Medicine (J.P.S.) is also acknowledged.

26. Arendt J (2012) Biological rhythms during residence in polar regions. Chronobiol Int 29(4):379-394.

27. Stuster J (2004) Lessons from previous expeditions for the human exploration of Mars. J Brit Interplanet Soc 57(3-4):126-134.

28. Kanas N, et al. (2009) Psychology and culture during long-duration space missions Acta Astronaut 64(7-8):659-677.

29. Palinkas LA, Suedfeld P (2008) Psychological effects of polar expeditions. Lancet 371(9607):153-163.

30. Leon GR, Sandal GM, Larsen E (2011) Human performance in polar environments. $J$ Environ Psychol 31(4):353-360.

31. Franken P, Chollet D, Tafti M (2001) The homeostatic regulation of sleep need is under genetic control. J Neurosci 21(8):2610-2621

32. Van Dongen HPA, Baynard MD, Maislin G, Dinges DF (2004) Systematic interindividual differences in neurobehavioral impairment from sleep loss: Evidence of trait-like differential vulnerability. Sleep 27(3):423-433.

33. Viola AU, et al. (2007) PER3 polymorphism predicts sleep structure and waking performance. Curr Biol 17(7):613-618.

34. Landolt HP (2008) Genotype-dependent differences in sleep, vigilance, and response to stimulants. Curr Pharm Des 14(32):3396-3407.

35. Goel N, Banks S, Mignot E, Dinges DF (2010) DQB1*0602 predicts interindividual differences in physiologic sleep, sleepiness, and fatigue. Neurology 75(17):1509-1519.

36. Dijk DJ, Archer SN (2010) PERIOD3, circadian phenotypes, and sleep homeostasis. Sleep Med Rev 14(3):151-160.

37. Kuna ST, et al. (2012) Heritability of performance deficit accumulation during acute sleep deprivation in twins. Sleep 35(9):1223-1233.

38. Czeisler CA (2011) Impact of sleepiness and sleep deficiency on public health-utility of biomarkers. J Clin Sleep Med 7(5, Suppl):S6-S8.

39. Goel N, Dinges DF (2012) Predicting risk in space: Genetic markers for differential vulnerability to sleep restriction. Acta Astronaut 77:207-213.

40. Siegel JM (2009) Sleep viewed as a state of adaptive inactivity. Nat Rev Neurosci 10(10):747-753.

41. Simpson JA, Weiner ESC, eds (1989) Oxford English Dictionary (Oxford Univ Press, New York), 2nd Ed.

42. Carey HV, Andrews MT, Martin SL (2003) Mammalian hibernation: Cellular and molecular responses to depressed metabolism and low temperature. Physiol Rev 83(4): 1153-1181.

43. Jones SG, Paletz EM, Obermeyer WH, Hannan CT, Benca RM (2010) Seasonal influences on sleep and executive function in the migratory White-crowned Sparrow (Zonotrichia leucophrys gambelii). BMC Neurosci 11:87.

44. Siegel JM (2008) Do all animals sleep? Trends Neurosci 31(4):208-213.

45. Scheer FAJL, Wright KP, Jr., Kronauer RE, Czeisler CA (2007) Plasticity of the intrinsic period of the human circadian timing system. PLOS ONE 2(8):e721.

46. Buxton OM, Lee CW, L'Hermite-Baleriaux M, Turek FW, Van Cauter E (2003) Exercise elicits phase shifts and acute alterations of melatonin that vary with circadian phase. Am J Physiol Regul Integr Comp Physiol 284(3):R714-R724.

47. Barger LK, et al. (2012) Learning to live on a Mars day: fatigue countermeasures during the Phoenix Mars Lander mission. Sleep 35(10):1423-1435.

48. Fu Q, et al. (2002) Cardiovascular and sympathetic neural responses to handgrip and cold pressor stimuli in humans before, during and after spaceflight. $J$ Physiol 544(Pt 2):653-664

49. Zayzafoon M, Meyers VE, McDonald JM (2005) Microgravity: The immune response and bone. Immunol Rev 208(1):267-280

50. Basner M, et al. (2007) American Time Use Survey: Sleep time and its relationship to waking activities. Sleep 30(9):1085-1095.

51. Basner M, Dinges DF (2009) Dubious bargain: Trading sleep for Leno and Letterman. Sleep 32(6):747-752.

52. Wittmann M, Dinich J, Merrow M, Roenneberg T (2006) Social jetlag: Misalignment of biological and social time. Chronobiol Int 23(1-2):497-509.

53. Kantermann T, Juda M, Merrow M, Roenneberg T (2007) The human circadian clock's seasonal adjustment is disrupted by daylight saving time. Curr Bio/ 17(22):1996-2000.

54. Roenneberg T, Allebrandt KV, Merrow M, Vetter C (2012) Social jetlag and obesity. Curr Biol 22(10):939-943.

55. Ancoli-Israel S, et al. (2003) The role of actigraphy in the study of sleep and circadian rhythms. Sleep 26(3):342-392.

56. Basner M, Dinges DF (2011) Maximizing sensitivity of the psychomotor vigilance test (PVT) to sleep loss. Sleep 34(5):581-591.

57. Abe T, et al. (2011) Detecting deteriorated vigilance using percentage of eyelid closure time during behavioral maintenance of wakefulness tests. Int J Psychophysiol 82(3):269-274. 